\title{
Análisis de los factores de emisión dinámicos para el vehículo Toyota Hi Lux con motor de Ciclo Otto S.I para altitudes superiores a los 2500 metros.
}

\author{
Analysis of the dynamic emission factors for the Toyota Hi Lux vehicle with \\ the Otto S.I Cycle engine for altitudes above 2500 meters.
}

Paúl Montúfar Paz. ${ }^{1}$, Celin Abad Padilla. ${ }^{2}$, Milton Israel Quinga. ${ }^{3}$ \& Andrea Viviana Razo Cifuentes. $^{4}$

Recibido: 20-02-2019 / Revisado: 28-02-2019 /Aceptado: 14-03-2019/ Publicado: 05-04-2019

\begin{abstract}
.
DOI: https://doi.org/10.33262/cienciadigital.v3i2.469

The aim of this paper is to carry out analyzes the emission factors for light vehicles that govern their operation based on the Otto cycle for gasoline engines under the calculation parameters of the CIMA thermodynamic model studied and developed by Dr. Ignacio Huertas in the Monterrey technology of Mexico at the CIMA Automotive Mechatronics Research Center. One of the major drawbacks when quantifying the environmental cost of automobile circulation is the ability to consider the specific geographic conditions of the area within the calculation and the issue becomes more critical in countries such as Ecuador where the driving cycle has not It has still been fully defined as in larger cities where the cycles and measurement protocols have already been studied and implemented at a high level of detail. Since 1970, the implementation of vehicular emission control programs (I / M) has begun, which has differentiated from the homologation tests carried out on new vehicles, which allow determining the impact of the journey with its determined maintenance on the emissions generated for the automotive.
\end{abstract}

1 Escuela Superior Politécnica de Chimborazo, Riobamba, Ecuador, paul.montufar@espoch.edu.ec

2 Escuela Superior Politécnica de Chimborazo, Riobamba, Ecuador, celin.padilla@espoch.edu.ec

3 Escuela Superior Politécnica de Chimborazo, Riobamba, Ecuador, milton.quinga@espoch.edu.ec

4 Escuela Superior Politécnica de Chimborazo, Riobamba, Ecuador, arazo@espoch.edu.ec 
Keywords: Evaporators - Production - Consumer products - Food industry.

\section{Resumen}

El presente estudio de investigación analiza los factores de emisión para vehículos livianos que rigen su operación en base al ciclo Otto para motores a gasolina bajo los parámetros de cálculo del modelo termodinámico CIMA estudiado y desarrollado por el doctor Ignacio Huertas en el tecnológico de Monterrey de México en el Centro de Investigación en Mecatrónica Automotriz CIMA.

Uno de los grandes inconvenientes al momento de cuantificar el costo ambiental de la circulación de los automóviles es el poder considerar dentro del cálculo las condiciones geográficas específicas de la zona y el tema se vuelve más crítico en países como Ecuador donde el ciclo de conducción no ha sido aún definido plenamente como en urbes de mayor tamaño donde los ciclos y los protocolos de medición han sido ya estudiados y puestos en marcha a un gran nivel de detalle.

A partir de 1970 ha iniciado la implementación de programas de control de emisiones vehiculares (I/M) que a diferencia de las pruebas de homologación que se realizan en vehículos nuevos, estas permiten determinar el impacto del recorrido con su determinado mantenimiento sobre las emisiones generadas por el automotor.

Palabras Claves: Evaporadores - Producción - Productos de consumo - Industria alimentaria.

\section{Introduccion.}

La contaminación atmosférica es la presencia en el aire de sustancias (partículas líquidas, solidas o gaseosas suspendidas) en cantidades que generan inconvenientes a la salud, los mismos que pueden ser primarios como: partículas suspendidas (PS), bióxido de azufre (SO2), dióxido de carbono (CO2), monóxido de carbono (CO), bióxido de nitrógeno (NO2), monóxido de nitrógeno (NO), hidrocarburos $(\mathrm{HC})$ y los secundarios que resultan de la combinación de los primeros. Durante el 2016 las emisiones globales producto del CO2 fueron de $32.31 \mathrm{Gt} \mathrm{CO} 2$, si bien los últimos 3 años se ha mantenido casi estable mirando hacia atrás, las emisiones se han duplicado desde principios de los años setenta y han aumentado alrededor de un $40 \%$ desde 2000. (IEA. , 2016)

El motor de combustión interna transforma la energía química contenida en el combustible para transformarla en energía cinética, capas de mover al automóvil, sin embargo en este proceso de combustión una cantidad importante de residuos se generán y mayor y menor medida dependiendo de la eficiencia con que la mezcla y reacción de los reactivos se lleve a cabo. (Montúfar, Caracterización del modelo termodinámico Otto y Cima para la predicción de potencia, torque y consumo de un motor de combustion interna S.I con el dinamometro automotriz para evalauar los resultados, 2018) 
Si bien una combustión completa genera solamente: dióxido de carbono, agua y nitrógeno este tipo de reacción en la práctica no sucede pues en el afán de lograr una proporción estequiométrica se recurre a un ciclo de lazo cerrado donde en base al sensor de oxígeno se corrige permanentemente la señal obteniendo fases de mezcla enriquecida y empobrecida a cada instante lo que genera una reacción incompleta de la combustión del combustible, situación que desencadena una serie de emisiones irregulares desencadenando las emisiones de HC y CO. (Montúfar, Caracterización del modelo termodinámico Otto y Cima para la predicción de potencia, torque y consumo de un motor de combustion interna S.I con el dinamometro automotriz para evalauar los resultados, 2018)

Ciclos de conducción como el NEDC ha sido considerando en los últimos años para evaluar el impacto ambiental de los automóviles en base a la legislación europea sin embargo la brecha existente entre los resultados generados por esta prueba bajo parámetros ideales de funcionamiento en laboratorios preparados para el efecto con variables controladas y los resultados arrojados en pruebas reales en carretera han levantado fuertes críticas a este ciclo de conducción. (TZIRAKIS, PITSAS, ZANNIKOS, \& STOURNAS, 2006)

Es por esto que el presente estudio toma como punto de partida las emisiones generadas en carretera bajo parámetros de conducción real conforme al patrón de conducción existente en ciudades del Ecuador y que se busca cotejar y determinar la correlación existente respecto al modelo CIMA estudiado en el ITESM en el Centro de Investigación en Mecatrónica Automotriz. (Montúfar, Caracterización del modelo termodinámico Otto y Cima para la predicción de potencia, torque y consumo de un motor de combustion interna S.I con el dinamometro automotriz para evalauar los resultados, 2018)

El impacto ambiental generado por los vehículos en Ecuador tiene un cierto grado de incertidumbre debido a que el ciclo de conducción propio de nuestra geografía está aún por definir lo cual ha planteado que los modelos a utilizar para dicho propósito sean importados y con variables extrañas a nuestra realidad vial.

Técnicas de medición en de los factores de emisión en laboratorio no consideran todas las variables que entran al juego durante la conducción real como es el caso del coeficiente real de rodadura entre los neumáticos y la calzada habitual de circulación, además la carga aerodinámica existente varía fuertemente en comparación con las pruebas controladas. Lo cual lleva a la hipótesis de que los factores de emisiones o masa contaminante generada por unidad de distancia de conducción será significativamente más grande.

\section{Importancia del Problema}

La conminación generada por las emisiones vehiculares ha sido clasificada en 3 grupos dependiendo del momento en que estas son generadas, siendo estas: emisiones en motor frío, emisiones durante la estabilidad térmica y las emisiones generadas por la evaporación del combustible. Para poder abordar esta situación las diferentes regiones del orbe han utilizado 
una serie de normas ambientales para poder evaluar los factores de emisión los mismos que están expresados por la siguiente ecuación:

$E T_{p=F E_{p} N A_{p} F C_{p}}(E c .1)$

Donde:

$E T_{p}:$ Emisiones totales $[\mathrm{gr}]$

$F E_{p}:$ Factor de emisiòn $\left[\frac{g r}{k m}\right]$

$N A_{p}:$ Nivel de actividad vehicular $[\mathrm{km}]$

$F C_{p}:$ Factor de corrección

Este parámetro de medición es cuantificado en base a un proceso de homologación en el cuál la marca del automotor se acoge a la normativa internacional. En el caso de la unión europea existe un circuito estándar llamado NEDC por sus siglas en ingles de nuevo ciclo de conducción europeo el mismo que este compuesto por 4 ciclos urbanos(ECE15) y un ciclo extraurbano (EUDC) con las características mostradas en el gráfico 1.

\section{Gráfico 1. Nuevo ciclo de conducción europeo, NEDC}

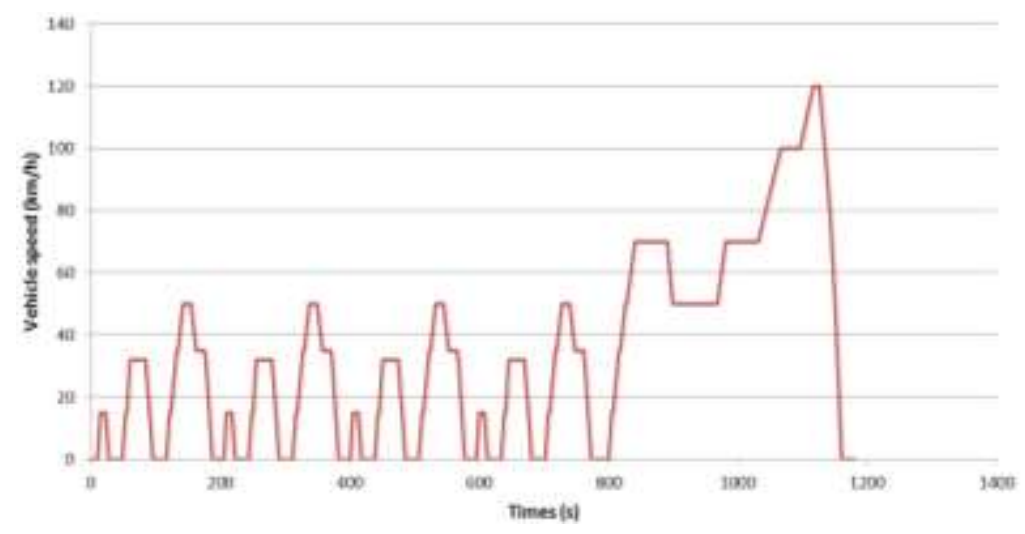

Fuente: Autor.

Estados unidos a través de EPA, Agencia de Protección Ambiental, regula las emisiones aplicando el ciclo de conducción FTP- 15, el mismo que consta de dos partes interurbanas una al inicio y otra al fin y en medio una parte urbana como lo muestra la gráfica 2.

\section{Gráfico 2, Ciclo de conducción FTP-15}




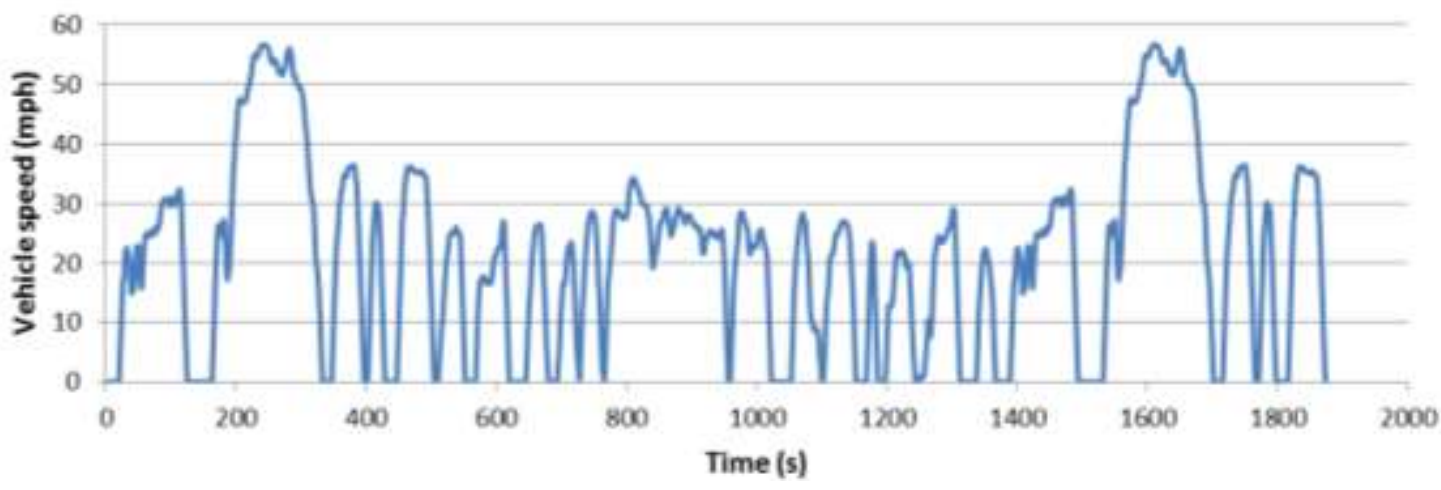

Fuente: Autor.

A partir de estos ciclos de conducción se ha regulado las emisiones generadas conforme a la norma europea para los autos nuevos vendidos en los Estados Miembros de la Unión Europea con implantación progresiva cada vez más restrictivas.

Tabla 1. Evolución de las normas EURO

\begin{tabular}{lllll}
\hline Normativa & CO & HC & NOx & NOx \\
\hline Euro 1 & 2,72 & & 0,95 & \\
Euro 2 & 2,2 & & 0,5 & \\
Euro 3 & 2,3 & 0,2 & & 0,15 \\
Euro 4 & 1 & 0,1 & & 0,08 \\
Euro 5 & 1 & 0,075 & & 0,06 \\
\hline
\end{tabular}

Fuente: Autor

Para el Ecuador la normativa de emisiones vehiculares establece los parámetros de monóxido de carbono y partes por millón de hidrocarburos conforme a la tabla 2. Para pruebas estáticas y conforme a la tabla 3 para dinámicas.

Tabla 2. Normas de límites de emisiones para el Ecuador en pruebas al vacío. 


\begin{tabular}{lllll}
\hline Año & \multicolumn{2}{l}{$\begin{array}{l}\text { Monóxido de carbono } \\
{[\%]}\end{array}$} & \multicolumn{2}{l}{ Hidrocarburos [ ppm] } \\
\cline { 2 - 5 } & $0 / 1500$ & $1500 / 300$ & $0 / 1500$ & $1500 / 300$ \\
$2000 \quad \mathrm{y}$ & 1 & 1 & 200 & 200 \\
posteriores & & & & \\
$\begin{array}{l}\text { de } 1990 \text { a } \\
1999\end{array}$ & 3,5 & 4,5 & 650 & 750 \\
$1989 \quad$ y & 5,5 & 6,5 & 1000 & 1200 \\
posteriores & & & &
\end{tabular}

Fuente: Autor

Tabla 3. Límites de emisiones para pruebas dinámicas para el Ecuador

\begin{tabular}{|c|c|c|c|c|c|c|c|}
\hline Categorla & \begin{tabular}{|c|} 
Pego \\
bruto dot \\
veniculo \\
$\mathrm{kg}$
\end{tabular} & $\begin{array}{c}\text { P9so del vehiculo } \\
\text { cargado } \\
\mathrm{kg}\end{array}$ & $\begin{array}{l}\mathrm{CO} \\
\mathrm{g} / \mathrm{km}\end{array}$ & $\begin{array}{l}\mathrm{HC} \\
\mathrm{g} \mathrm{kmm}\end{array}$ & $\begin{array}{l}\text { NOX } \\
\text { g/km }\end{array}$ & $\begin{array}{c}\text { CICLOS DE } \\
\text { PRUEBA }\end{array}$ & $\begin{array}{c}\text { Evaporativas } \\
\text { g/enasyo } \\
\text { SHED }\end{array}$ \\
\hline Vehiculos Liviances & & & 2,10 & 0,25 & 0,62 & \multirow[t]{3}{*}{ FTP-75 } & 2 \\
\hline \multirow{2}{*}{\begin{tabular}{|l|} 
Vohiculios \\
Merimos
\end{tabular}} & \multirow[t]{2}{*}{$=<3860$} & $=<1700$ & 6.2 & 0,5 & 0.75 & & 2 \\
\hline & & $1700-3860$ & 6.2 & 0,5 & 1,1 & & 2 \\
\hline \multirow[t]{2}{*}{\begin{tabular}{|l|} 
Vehiculios \\
Pesados"
\end{tabular}} & $\begin{array}{l}>3860= \\
<6350\end{array}$ & & 14.4 & 1,1 & 5.0 & \multirow[t]{2}{*}{$\begin{array}{c}\text { Trensilente } \\
\text { pessido }\end{array}$} & 3 \\
\hline & $>6350$ & & 37,1 & 1,9 & 5,0 & & 4 \\
\hline
\end{tabular}

Fuente: INEN 2204:2002

Este protocolo de medición no se ajusta a la situación geográfica de las ciudades de Ecuador en vista de que no se acoge a un ciclo de conducción real ajustado a nuestros patrones de conducción que involucran: infraestructura vial actualizada, tecnologías vehiculares, trafico presente, desnivel de las carreteras y modos de conducción entre otros por lo cual el parámetro de medición en pruebas varía notablemente conforme a las emisiones reales en carretera y ciudad.

\section{Metodología}


Como se mencionó en la introducción, el presente estudio busca correlacionar un método teórico de cálculo de factores de emisiones con una lectura real de las mismas en carretera bajo un ciclo de conducción que se adapte las condiciones geográficas y viales propias de nuestro país, para tal efecto la metodología de medición se enfocará en las con emisiones generadas en el periodo de calentamiento del automóvil, (E partida) en ralentí y principalmente durante el lapso en que el automóvil se encuentra en su temperatura de funcionamiento (E caliente ). (Alvarado \& Aguirre, 2000)

\section{Equipos y materiales}

\section{Analizador de emisiones Maha Met 6.3}

Para la obtención de lecturas de emisiones se cuenta con un analizador de emisiones Maha Met 6.3 el cuál permitió obtener las lecturas de emisiones de monóxido de carbono, dióxido de carbono, oxígeno y los hidrocarburos, el equipo tiene las especificaciones mostradas en la tabla 4.

El equipo permitió obtener las lecturas de gases en las fases de precalentamiento y a temperatura normal de funcionamiento.

Tabla 4. Especificaciones técnicas Maha Met 6.3

\begin{tabular}{|c|c|}
\hline Gases mesurables & $\mathrm{HC}, \mathrm{CO}, \mathrm{CO} 2, \mathrm{O} 2$ \\
\hline Principio de medición espectrometría de infrarrojo & $\mathrm{HC}, \mathrm{CO}, \mathrm{CO} 2$ \\
\hline Principio de medición detección electroquímica & $\mathrm{O} 2$ \\
\hline Índice de flujo & $3.51 / \mathrm{min}$ \\
\hline Clase de precisión & $\mathrm{O}(\mathrm{OIML})$ \\
\hline Tensión de a bordo & $10 \mathrm{~V} / 30 \mathrm{~V}$ \\
\hline Alimentación de corriente & 1/N/PE $110 \mathrm{~V} / 230 \mathrm{~V} 50 \mathrm{~Hz} / 60 \mathrm{~Hz}$ \\
\hline Temperatura ambiente & $0^{\circ} \mathrm{C}-45^{\circ} \mathrm{C}$ \\
\hline Altura de funcionamiento & $-100 \mathrm{~mm}-3000 \mathrm{~mm}$ \\
\hline Dimensiones totales ( $\mathrm{La} \times \mathrm{An} \times \mathrm{Al})$ & $406 \mathrm{~mm}$ x $225 \mathrm{~mm}$ x $160 \mathrm{~mm}$ \\
\hline Peso & $4.6 \mathrm{~kg}$ \\
\hline CO - Rango de medición/Exactitud de medición (máx) & - $15 \%$ Vol. / 0,01 \\
\hline $\begin{array}{l}\text { CO2 - Rango de medición/Exactitud de medición } \\
\text { (máx) }\end{array}$ & $-20 \%$ Vol. / 0,01 \\
\hline HC - Rango de medición/Exactitud de medición (máx) & - 9999 ppm / 0,1 \\
\hline O2 - Rango de medición/Exactitud de medición (máx) & $-25 \%$ Vol. / 0,01 \\
\hline Lambda (calculada) & $0,5-9,99 / 0,01$ \\
\hline Principio de medición & Extinción \\
\hline $\begin{array}{l}\text { Tiempo de calentamiento aprox. de la célula de } \\
\text { medición }\end{array}$ & $150 \mathrm{~s}$ \\
\hline
\end{tabular}


Vol. 3, N², p. 602-619, abril - junio, 2019

Tensión de a bordo

Alimentación de corriente

Dimensiones totales ( $\mathrm{La} \times \mathrm{An} \times \mathrm{Al}$ )

Peso

Rango de medición concentración de partículas

Resolución concentración de partículas

Intervalo de medición opacidad

Área de medición coeficiente de absorción

Resolución coeficiente de absorción
$10 \mathrm{~V} / 30 \mathrm{~V}$

1/N/PE $110 \mathrm{~V} / 230 \mathrm{~V} 50 \mathrm{~Hz} / 60 \mathrm{~Hz}$

$406 \mathrm{~mm}$ x $225 \mathrm{~mm}$ x $160 \mathrm{~mm}$

$4.6 \mathrm{~kg}$

- $1100 \mathrm{mg} / \mathrm{m}^{3}$

$1 \mathrm{mg} / \mathrm{m}^{3}$

$-100 \%$

$-9.99 \mathrm{~m}-1$

$0.01 \mathrm{~m}-1$

Fuente.- Página oficial Maha

\section{Módulo de Interfaz CAN de la Serie C NI-9862}

El NI-9862 es una interfaz de Red de Controladores de Área (CAN) de Razón de Datos Flexible (FD) de alta velocidad para desarrollar aplicaciones con el controlador NI-XNET.

El NI-9862 funciona bien en aplicaciones que requieren manipulación de alta velocidad en tiempo real de cientos de marcos y señales CAN, como simulación de hardware-in-the-loop, rápida generación de prototipos de control, monitoreo de bus, control de automatización y más.

A fin de poder obtener lecturas en el orden de los milisegundos se utilizó esta tarjeta de adquisición de datos para poder correlacionar las lecturas obtenidas por el Maha Met 6.3 con los parámetros de funcionamiento obtenidos de la Red CAN - BUS del automóvil.

Tabla 5. Especificaciones NI 9862

\begin{tabular}{ll}
\hline Característica & Valor \\
\hline & $95 \Omega$ min \\
Impedancia & $120 \Omega$ nominal \\
& $140 \Omega$ Max \\
Retardo de $\quad$ línea & $5 \mathrm{~ns} / \mathrm{m}$ \\
nominal & NXP \\
Transceptor & TJA1041AT \\
Max baud radio & 1 Mbps \\
CAN H, CAN L bus & $27 / 40$ VDC
\end{tabular}


Fuente de Voltaje CAN 9 a 30 VDC

Peso

$144 \mathrm{~g}$

Fuente. - Página oficial National Instrumets

\section{Ciclo de conducción}

A partir del análisis de las velocidades recurrentes en diferentes ciudades del Ecuador se estableció que las velocidades a las que normalmente un vehículo se desplaza en nuestras vías de ciudades andinas con desniveles considerables y un tráfico moderado son las que se exponen en el gráfico 3 al mismo que llamare Ciclo de conducción andino PTEC-593. El ciclo considera dos fases de circulación en ciudad a velocidades moderadas entre 40 y 50 $\mathrm{km} / \mathrm{h}$ y una fase final de carretera con menos aceleraciones y velocidades de punta de hasta $90 \mathrm{~km} / \mathrm{h}$.

Gráfico 3. Ciclo de conducción PTEC-593

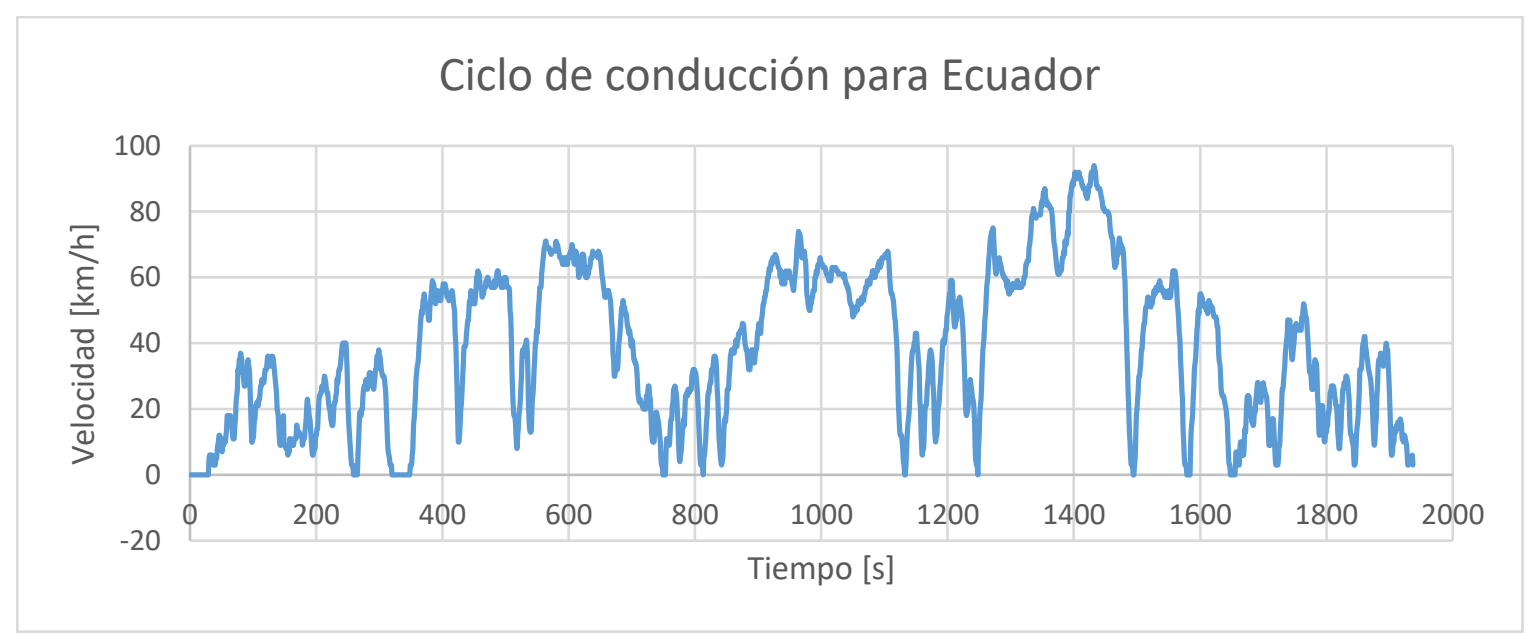

A partir del analizador de emisiones MAHA Met 6.3 y la tarjeta de adquisición de datos $\mathrm{Ni}$ 9862 se instrumentaron los automóviles para poder obtener las lecturas de emisiones (CO, $\mathrm{HC}, \mathrm{CO} 2$, Lambda y O2) y los parámetros de funcionamiento del automóvil (Sensores de oxígeno de banda ancha, sensores de posición del acelerador TPS, sensor de flujo de masa de aire MAF, sensores de temperatura ECT y IAT, sensor de revoluciones CKP, sensor de velocidad del automóvil VSS.

Protocolo de acondicionamiento previo a la medición de emisiones 
El protocolo de medición toma como referencia la Norma INEN 2203 para revisiones vehiculares en condición de vacío en lo concerniente a pruebas estáticas donde dicho proceso se lo realiza de la siguiente manera:

- Antes de la prueba, realizar las verificaciones siguientes:

- Someter al equipo a un período de calentamiento y estabilización, según las especificaciones del fabricante.

- Retirar todo material en forma de partículas y eliminar toda substancia extraña o agua, que se hayan acumulado en la sonda de prueba y que puedan alterar las lecturas de la muestra.

- Revisar que la transmisión del vehículo esté en neutro (transmisión manual) o parqueo (transmisión automática).

- Revisar que el control manual del ahogador (choque), no se encuentre en operación, y que los accesorios del vehículo (luces, aire acondicionado, etc.), estén apagados.

- Revisar en el vehículo que el sistema de escape se encuentre en perfectas condiciones de funcionamiento y sin ninguna salida adicional a las del diseño que provoque dilución de los gases de escape o fugas de los mismos. Las salidas adicionales a las contempladas en el diseño original no deben ser aceptadas, aunque éstas se encuentren bloqueadas al momento de la prueba.

- Revisar que el nivel de aceite en el cárter esté entre el mínimo y máximo recomendado por el fabricante, con el motor apagado y el vehículo en posición horizontal.

- Encender el motor del vehículo y verificar que se encuentre a la temperatura normal de operación.

\section{Medición en vacío}

- Conectar el tacómetro del equipo de medición al sistema de encendido del motor y verificar las condiciones de marcha mínima o "ralentí".

- Con el motor a temperatura normal de operación y en condición de marcha mínima o "ralentí", introducir la sonda de prueba en el punto de salida del sistema de escape del vehículo. Tener la seguridad de que la sonda permanezca fija dentro del sistema de escape mientras dure la prueba.

- Esperar el tiempo de respuesta del equipo de medición dado por cada fabricante.

- Imprimir las lecturas estabilizadas de las emisiones medidas.

- Si, por diseño, el vehículo tiene doble sistema de escape, medir por separado cada salida. El valor del resultado final será la mayor lectura registrada.

\section{Protocolo de medición de emisiones dinámicas a temperatura normal de funcionamiento.}


- Comprobar que el motor haya llegado a su temperatura de funcionamiento a través de la lectura del ECT.

- Realizar el ciclo de conducción en carretera PTEC-593 procurando ceñirse con una margen no superior al $3 \%$ de variación respecto a factores comunes como la velocidad y tiempos de prueba a fin de asegurar la reproducibilidad y repetitividad del ciclo.

- Utilizar el analizador de emisiones Maha Met 6.3 para recolectar las lecturas de emisiones y la tarjeta de adquisición de datos NI 9862 para correlacionar con los datos de operación del automóvil recolectados a partir de la red CAN bus del automóvil.

- Las pruebas se las realizaron en vehículos que operan bajo el Ciclo Otto de diferentes marcas y cilindradas de 1600 cc, 2000 cc y 2700 cc con inyección directa e indirecta de combustible.

- Registrar los datos de emisiones y sensores de operación del automóvil.

\section{Resultados}

A partir de las 1842 lecturas obtenidas para el ciclo de conducción PTEC-593 se obtuvieron las lecturas del analizador de emisiones Met 6.3 y de la instrumentación de los sensores del vehículo con la tarjeta de adquisición de datos National Instrument NI 9862 .

Señales:

MET 6.3

1. Monóxido de carbono.

2. Dióxido de carbono.

3. Hidrocarburos.

4. Lambda.

5. Oxígeno.

\section{NI 9862}

1. Angulo de pendiente.

2. Sensor de flujo de aire.

3. Sensor de oxígeno.

4. Velocidad.

5. Radio de mezcla.

6. Velocidad de motor.

7. Apertura de mariposa de aceleración.

Los promedios de las lecturas obtenidas por el analizador de emisiones se muestran en la tabla 6, a partir de esas lecturas y utilizando el sensor de oxígeno de banda ancha se determino el consumo de combustible por unidad de distancia lo cuál se cotejo con la ecuación de productos y reactivos en la cámara de combustión, conforme a la ecuación 2 , donde $a, b, c, d, e, f$ 
$y k$ son los coeficientes estequiométricos desconocidos que se pueden calcular en base a los datos obtenidos por el analizador de emisiones. (Montúfar P. , 2018)

$\mathrm{CH}_{y}+k\left(0.21 \mathrm{O}_{2}+0.79 \mathrm{~N}_{2} \rightarrow a C O+b \mathrm{H}_{2} \mathrm{O}+c \mathrm{C}_{3} \mathrm{H}_{6}+d \mathrm{CO}_{2}+e \mathrm{~N}_{2}+\mathrm{FNO}\right.$ (Ec. 2)

Tabla 6. Emisiones generadas en la prueba dinámica en Ruta en el ciclo de conducción MET 6.3

Fuente. - Autor

\begin{tabular}{lll}
\hline \multicolumn{4}{c}{ Valores de emisiones } \\
\hline Monóxido de carbono [CO] & 0,37 & {$[\%]$} \\
Dióxido de carbono [CO2] & 11,09 & {$[\%]$} \\
Hidrocarburos [C3H6] & 19,337 & {$[\mathrm{ppm}]$} \\
Lambda & 1,57 & \\
Oxígeno [O2] & 2,7572 & {$[\mathrm{ppm}]$} \\
\hline
\end{tabular}

Considerando que las lecturas del analizador proporcionan la concentración volumétrica de los gases presentes en el escape sin considerar al vapor de agua se procede a calcular los factores de emisión del monóxido de carbono y de los hidrocarburos con la media de datos obtenidos en el ciclo de conducción preestablecido.

Gráfico 4.- Concentración de Monóxido de Carbono vs. Tiempo para el ciclo de conducción PTEC-593

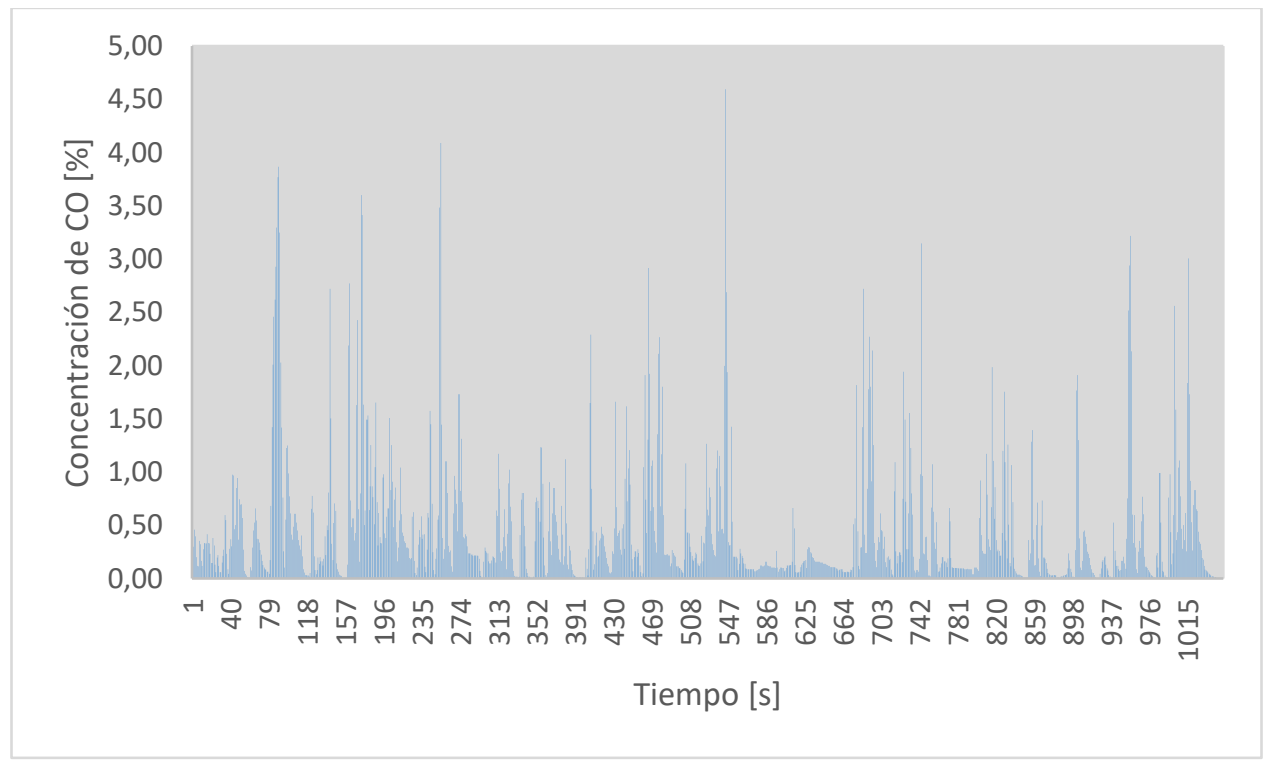

Fuente: Autor

Conforme al análisis de el gráfico 5 se puede apreciar que la concentración de dióxido de carbono permaneció prácticamente estable con valores cercanos al 15.2\% evidenciando el buen estado del automóvil lo cuál proporciona la confiabilidad sobre las lecturas obtenidas. 
Gráfico 5.- Concentración volumétrica del dióxido de carbono

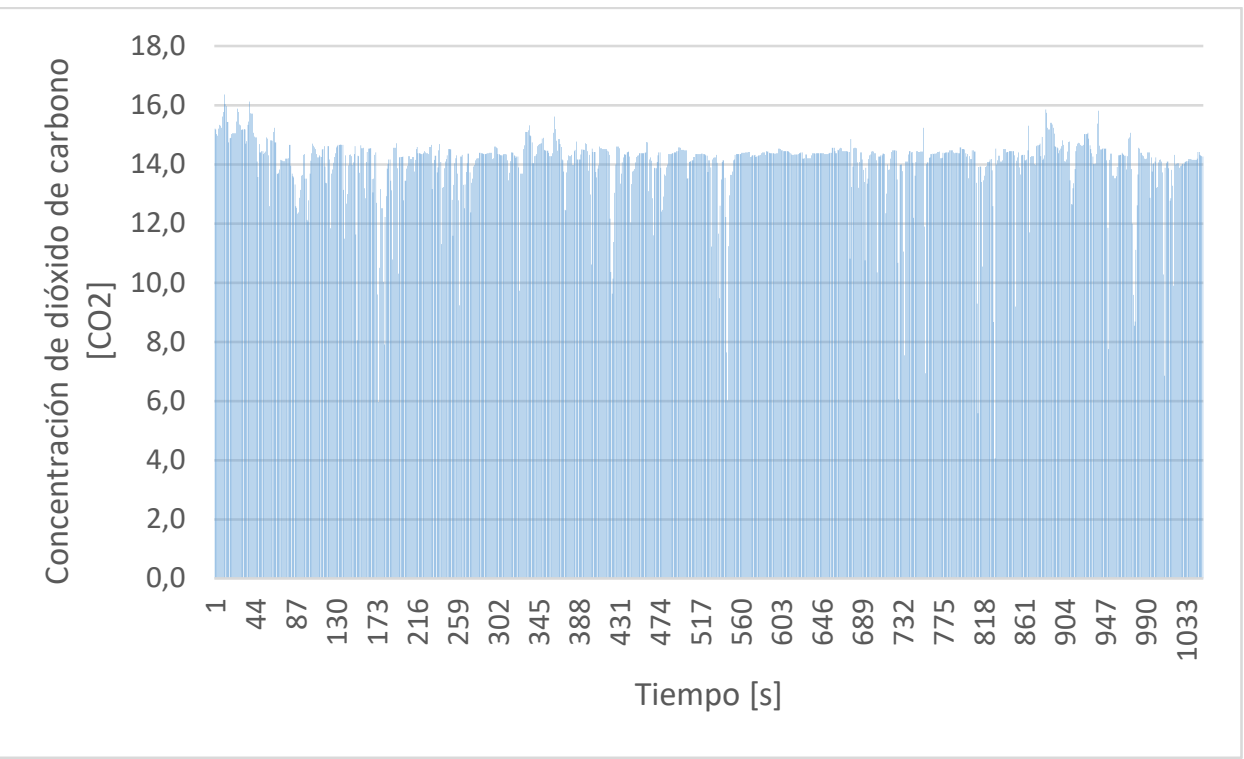

Fuente: Autor

En la gráfica 6 se puede ver además la concentración de Hidrocarburos respecto a la medición de tiempo, se determinó además la influencia de la pendiente, la velocidad, la relación aire combustible y la carga del motor sobre la cantidad de hidrocarburos sin quemar, lo cuál por medio de un análisis de regresión fue posible determinar, gráfica 7.

Gráfico 6. - Concentración de hidrocarburos en la prueba dinámica

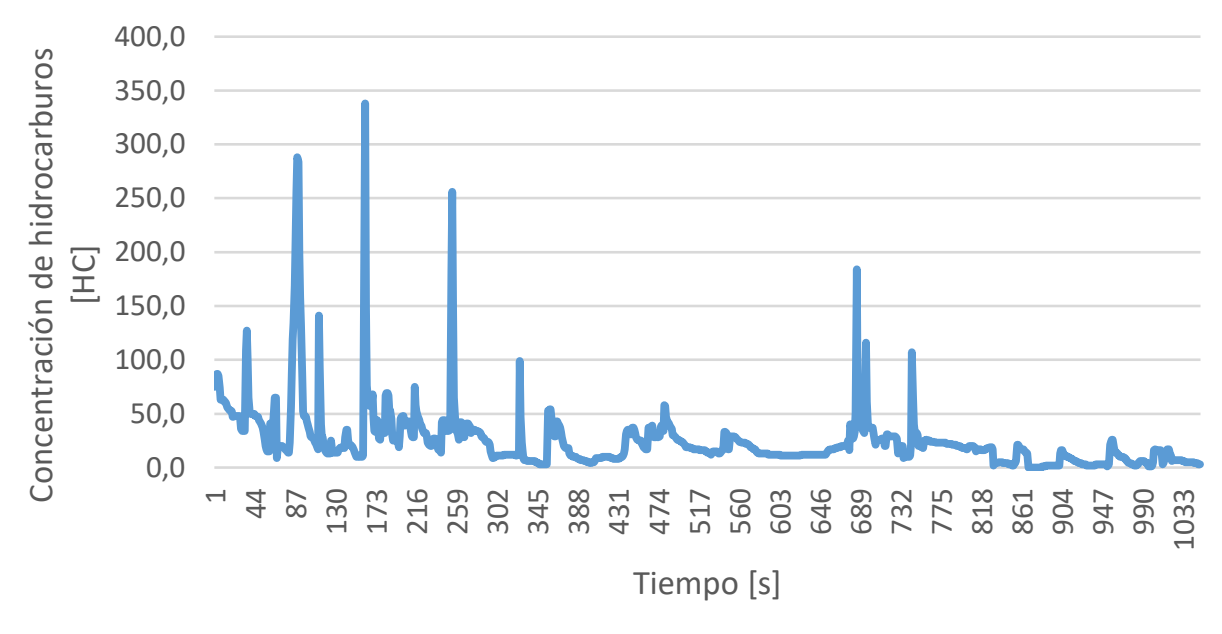

Fuente: Autor 
Gráfico 7. - Efectos principales de diferentes factores de desempeño del vehículo sobre el valor de $\mathrm{R}$ de correlación con la generación de hidrocarburos

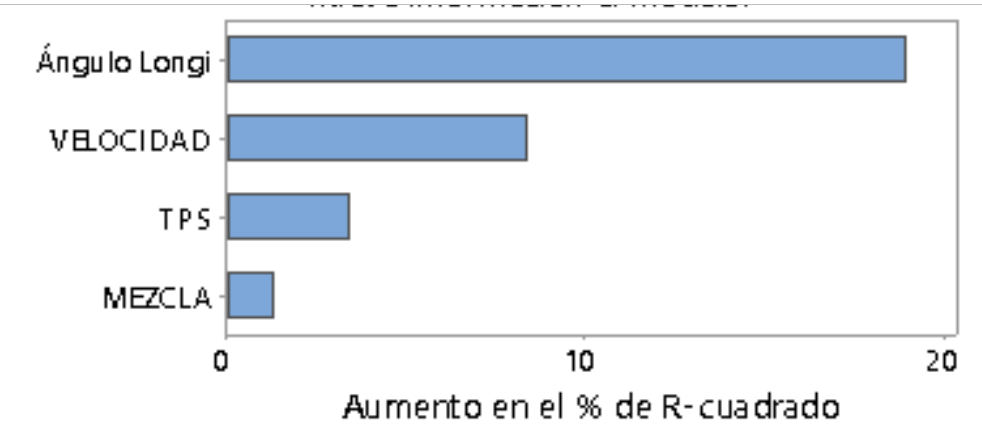

Fuente: Autor

Gráfico 8. - Análisis del efecto de variables operativas del vehículo sobre la concentración de hidrocarburos

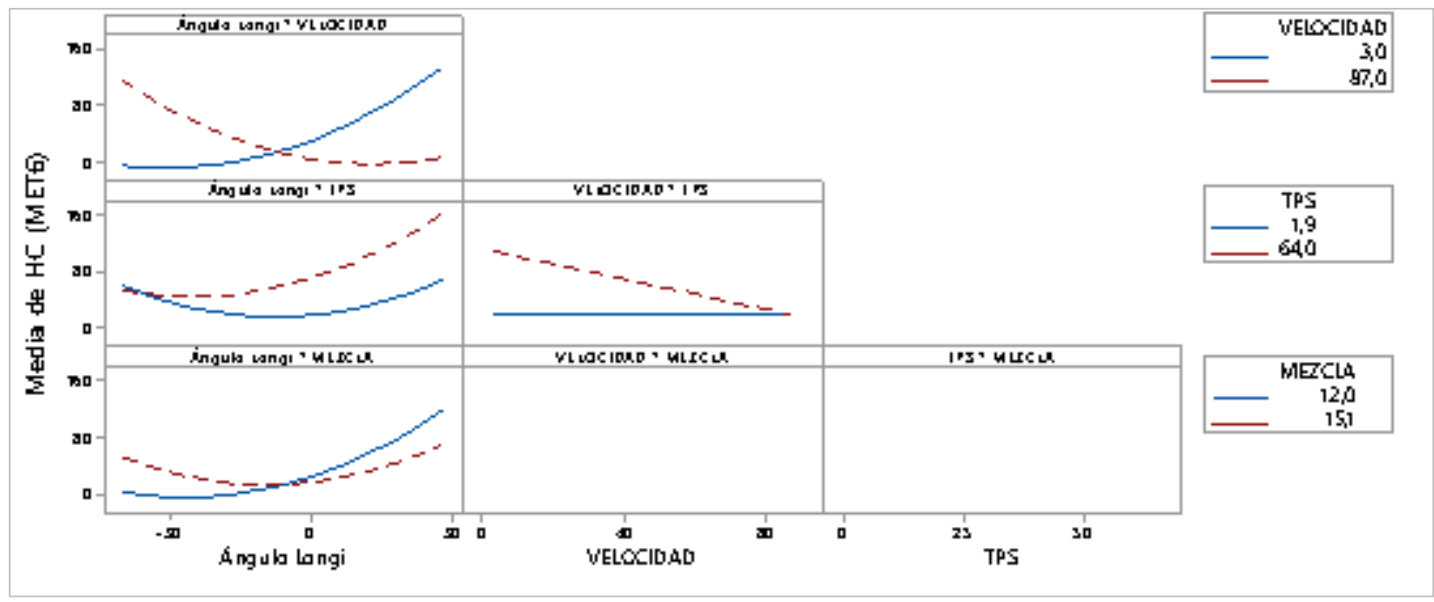

Fuente: Autor

A partir del análisis de la gráfica 8 se puede además concluir sobre el efecto de ciertas variables como la pendiente y velocidad sobre los hidrocarburos generados, determinando que sobre $80 \mathrm{~km} / \mathrm{h}$ la concentración de hidrocarburos al escape aumentan pendiente abajo el automóvil mientras que el acelerador a fondo cuando la velocidad es baja puede incrementar la lectura de hidrocarburos hasta las 160 ppm cuando con un adecuado tipo de conducción las emisiones son cercanas a la 0 ppm. 
Gráfico 9. - Análisis del efecto de variables operativas del vehículo sobre la concentración de monóxido de carbono.

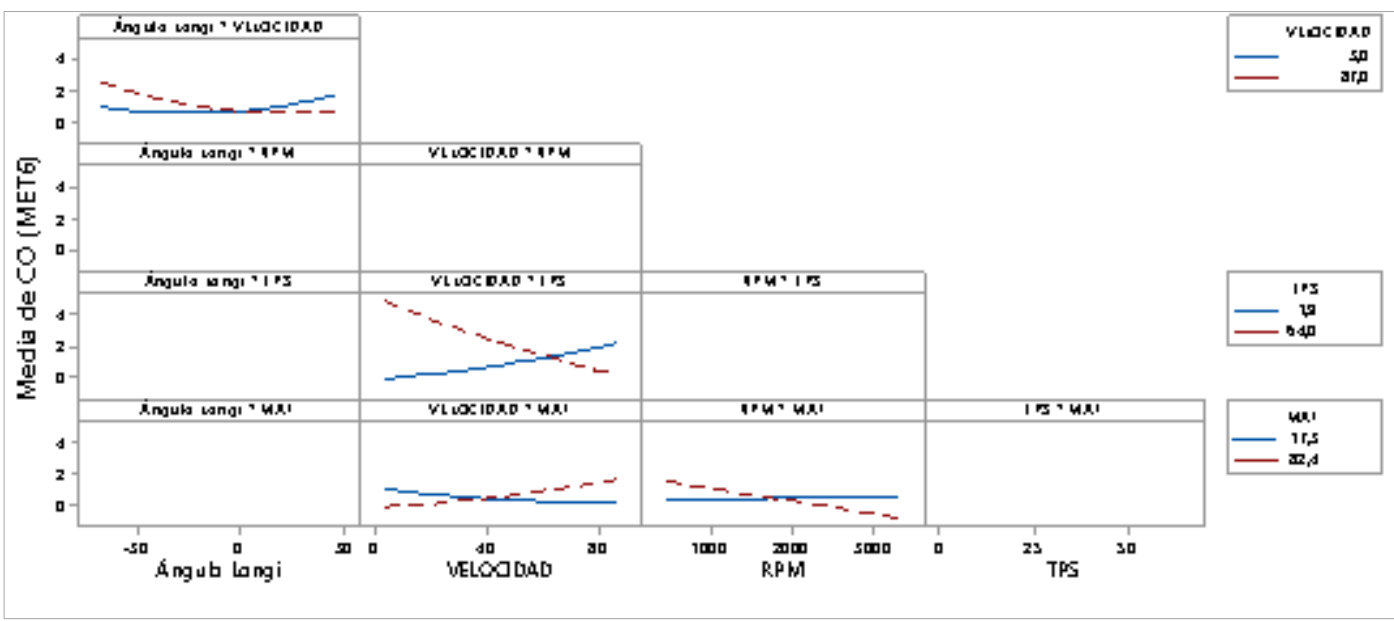

El análisis de la concentración del monóxido de carbono evidencia que la generación de emisiones tóxicas se incrementa principalmente en bajas velocidades con un TPS a máxima señal.

Considerando las lecturas de porcentaje volumétrico obtenido por el analizador de gases Maha MET 6.3 el cuál arroja los resultados de concentración volumétrica de:

- Dióxido de carbono.

- Monóxido de carbono.

- Hidrocarburos.

- Oxígeno.

- Óxidos de nitrógeno.

Con estos resultados una vez emparejados con el registro de los sensores de operación monitoreados por la tarjeta NI 9862 de National Instruments entre los cuales se encontraba el de velocidad, ángulo de pendiente, lectura de Maf y relación aire combustible permitieron determinar los factores de emisión del: monóxido de carbono y de hidrocarburos en una prueba dinámica bajo el ciclo de conducción PTEC-593.

Está información permitió evaluar la generación de emisiones en función de la distancia recorrida considerando que la concentración volumétrica leída por el analizador se encuentra en base seca y sin la participación de nitrógeno en concentración pura, por lo cuál es necesario aislar a estos componentes para determinar la concentración em el total de residuos de la combustión. (Kuhler \& Karstens, 1978)

Los factores de emisión obtenidas para el vehículo C fueron los siguientes: 
Tabla 7. - Factores de emisión vehículo Toyota Hi Lux 4x2 Cd 2016

\begin{tabular}{lc}
\hline Factores de emisión en Ruta \\
\hline \multicolumn{2}{c}{$\left[\frac{\mathrm{g}}{\mathrm{Km}}\right]$} \\
Monóxido de Carbono & Hidrocarburos \\
\multicolumn{3}{c}{$F^{\prime}[\mathrm{CO}]$} & $F^{\prime}[\mathrm{HC}]$ \\
20.34 & 0.19 \\
\hline
\end{tabular}

Fuente: Autor

\section{Conclusiones.}

- La metodología de cálculo implementada en el presente estudio permite obtener los factores de emisión a partir de la información vial que representa la actividad vehicular en zonas urbanas de altitud cercana a los $3000 \mathrm{msnm}$.

- A partir de los resultados se logro determinar que la camioneta Toyota Hi Lux 4x2 Cd 2016 generó un factor de 20.34 g/km bajo el ciclo de conducción PTEC-593, el cuál caracteriza el patrón de conducción vial en ciudades del Ecuador con altitudes cercanas a los $3000 \mathrm{msnm}$. Lo cuál se mantiene dentro del registro normal de emisiones para esa altitud respecto a los vehículos sedan estudiados anteriormente.

- Referente a la generación de hidrocarburos se apreció una evidente disminución respecto a vehículos semejantes puesto que la lectura normal a esta altitud está ente 1.12 y 1.13 mientras que la eficiencia de la combustión y su sistema de alimentación permite que este automóvil genere $0.19 \mathrm{ppm}$ muy inferior a los datos obtenidos en otros automóviles. (Bosch, 2009)

- El tipo de conducción es un factor determinante sobre la cantidad de residuos de hidrocarburos sin quemar y de monóxido de carbono, velocidades bajas con presión del acelerador brusca es el factor determinante al momento de generar una combustión incompleta con los inconvenientes que esto genera.

- Revisando el estado del arte se evidencia que la generación de hidrocarburos y monóxido de carbono se incrementa fuertemente con la altura, sin embargo la tecnología de los motores de Toyota logra reducir la generación de hidrocarburos debido a su perfecta dosificación de combustible con sensores de oxígeno de banda ancha que permiten obtener relaciones de mezcla extremadamente pobre en momentos de baja carga. 


\section{Referencias bibliográficas.}

Alvarado, M., \& Aguirre, A. (2000). Modelo de emisiones vehículares Modem. Revista de la Universidad Sntiago de Chile.

Bosch. (2009). Catálogo Sonda Lambda. Sensor de oxígeno.

IEA. - (2016). IEA, International Energy Agency. Obtenido de https://www.iea.org/statistics/co2emissions/

Kuhler, M., \& Karstens, D. (1978). Improved Driving Cycle for Testing Automotive Exhaust Emissions. SAE.

Montúfar. (2018). Análisis de emisiones de óxido de nitrogeno de un motor de ciclo Otto a partir del comportamiento de la relación Aire - Combustible y del avance de encendido. Ciencia Digital.

Montúfar. (2018). Caracterización del modelo termodinámico Otto y Cima para la predicción de potencia, torque y consumo de un motor de combustion interna S.I con el dinamometro automotriz para evalauar los resultados. Revista Ciencia Digital.

Montúfar, P. (2018). Análisis de emisiones de NOx de un motor S.I a partir de la relación aire combustible y del avance de encendido. Ciencia Digital.

TZIRAKIS, E., PITSAS, K., ZANNIKOS, F., \& STOURNAS, S. (2006). VEHICLE EMISSIONS AND DRIVING CYCLES: COMPARISON OF THE ATHENS DRIVING CYCLE (ADC) WITH ECE-15 AND EUROPEAN DRIVING CYCLE (EDC) . GLOBAL NEST.

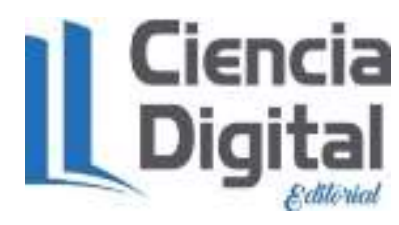




\section{PARA CITAR EL ARTÍCULO INDEXADO.}

Montúfar P., Abad C., Israel M. \& Razo A. (2019). Análisis de los factores de emisión dinámicos para el vehículo Toyota Hi Lux con motor de Ciclo Otto S.I para altitudes superiores a los 2500 metros. Revista electrónica Ciencia Digital 3(2), 602-619. Recuperado desde:

http://cienciadigital.org/revistacienciadigital2/index.php/CienciaDigital/article/view/469/1072

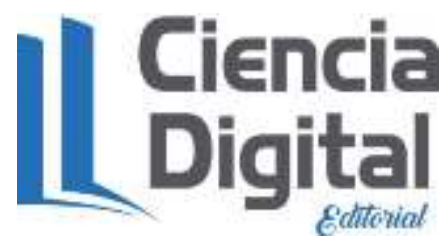

El artículo que se publica es de exclusiva responsabilidad de los autores y no necesariamente reflejan el pensamiento de la Revista Ciencia Digital.

El artículo queda en propiedad de la revista y, por tanto, su publicación parcial y/o total en otro medio tiene que ser autorizado por el director de la Revista Ciencia Digital.
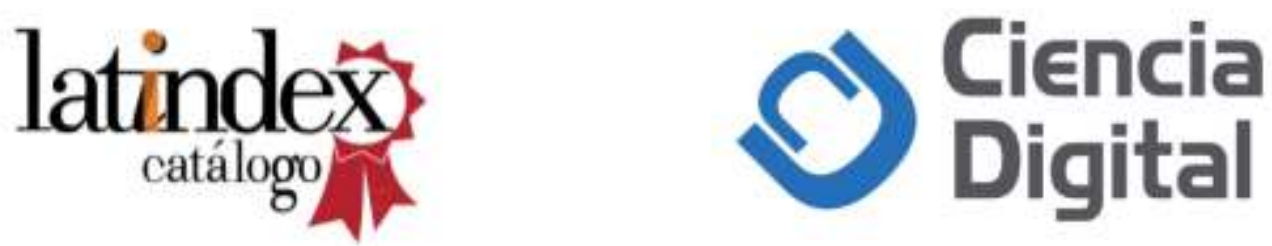